\title{
Successful Management of Abdominal Wound Dehiscence with Bogota bag, Vacuum assisted Closure combined with Tension Sutures
}

\author{
Abeysinghe AHMGB ${ }^{1}$, Senarathne $\mathbf{R}^{2}$ and Wimalasena GADNB ${ }^{3}$ \\ ${ }^{1}$ Consultant surgeon (MBBS, MS, FCSSL), Teaching Hospital Kuliyapitiya, Sri Lanka. \\ ${ }^{2}$ Research Assistant (MBBS), Department of Community Medicine, Faculty of Medicine, University of Peradeniya, Sri Lanka. \\ ${ }^{3}$ Medical Officer (MBBS), Teaching Hospital Kuliyapitiya, Sri Lanka.
}

*Corresponding Author: Abeysinghe AHMGB, Consultant surgeon (MBBS, MS, FCSSL), Teaching Hospital Kuliyapitiya, Sri Lanka.

Received date: August 08, 2021: Accepted date: September 24 2021: Published date: October 06, 2021

Citation: Abeysinghe AHMGB, Senarathne R., Wimalasena GADNB (2021) Successful Management of Abdominal Wound Dehiscence with Bogota bag, Vacuum assisted Closure combined with Tension Sutures. J. sur cas rep and img 4(7); DOI: 10.31579/2690-1897/091

Copyright: (C) 2021 Abeysinghe AHMGB. This is an open access article distributed under the Creative Commons Attribution License, which permits unrestricted use, distribution, and reproduction in any medium, provided the original work is properly cite.

\begin{abstract}
The burst abdomen management has advanced significantly. Here we present a management of a burst abdomen of morbid obese patient with combination of modality including Bogota bag, vacuum assisted closure and tension suturing. The patient underwent laparotomy for removal of sigmoid tumor with local infiltration and had wound dehiscence associated with infection, tissue oedema and necrosis. As patient was morbidly obese and had large wound gap, we decided to manage it with Bogota bag principal and Vacuum Assisted Closing. After successful formation of granulation tissue, we were able to apply tension sutures. Hence using combination of above-mentioned options, the patient was successfully sent back to his normal routines without any complication.
\end{abstract}

Key Words: abdominal wound dehiscence; bogota bag; burst abdomen; vacuum assisted closure; tension suturing

\section{Introduction}

Dehiscence is a partial or total separation of previously approximated wound edges due to failure of proper wound healing. The incidence of the event as described in international data ranging from $0.4 \%$ to $3.5 \%$ [1, 2]. The reported mortality rate is as high as $45 \%$ [3]. This scenario usually occurs between $5^{\text {th }}$ and $14^{\text {th }}$ day of the postoperative period ${ }^{4}$. But it can happen at any time in the postoperative period up to 30 days. Burst abdomen is seen more commonly in males over 60 years [5].

Early identification of wound dehiscence and extent of dehiscence (partial/full thickness) is very important. In most of the patients' disruption is warned by the appearance of serosanguinous discharge on the dressing [6]. If it happens before the $7^{\text {th }}$ day it may be considered pathognomonic of dehiscence ${ }^{6}$. There are various methods of surgical intervention to address the burst abdomen which include tension suturing, mesh repair, Bogota bag and vacuum assisted closure. These methods should be tailor made to the patient.

\section{Case presentation}

A 62 years $93 \mathrm{~kg}$ obese patient (Height $1.6 \mathrm{~m}, \mathrm{BMI}-36.32$ ) was referred from medical unit with an abdominal mass. He has had altered bowel habits for two months. He also complained of mucous diarrhea and on and off altered blood mixed with stool. He has no significant weight loss and appetite was normal. The colonoscopy and contrast enhance CT abdomen and pelvis revealed a sigmoid colonic tumor. His comorbidity includes type 2 diabetes mellitus and high blood pressure. After explaining to the patient, it was planned to proceed with elective sigmoid colectomy with optimizing his medical problem.

Laparotomy was performed under general anesthesia (5/5/2021) with all aseptic precautions. His bowel was prepared with polyethylene glycol the day before the surgery. During the procedure it was noted that a sigmoid tumor was adhered to the bladder dorm and loops of small intestine. The tumor was separated from the bladder and sigmoid colectomy was done incorporating a non-separable segment of small bowel loop. Colonic and small intestine anastomosis were done. Extreme care was taken not to spill intestinal content. Following surgery, patient was admitted to the ICU and managed there for 4 days and back in general word thereafter (patients vitals were normal during the perioperative period). On the $6^{\text {th }}$ postoperative day patient complained pain and mild discharge at the lower end of the abdominal wound. Other than that, he had no other constitutional symptoms. On inspection serous discharge was noted. Hence a couple of sutures were taken off. After the swab was taken for culture and for antibiotic sensitivity tests, cefuroxime and metronidazole intravenously were continued in microbiologists' opinion. On the $11^{\text {th }}$ postoperative day, it was noted that there was more discharge and it was smelly. Due to the very thick fat layer (nearly $12 \mathrm{~cm}$ ) of the patient it was 
difficult to decide whether the patient had a full thickness burst or not. Therefore, it was decided to take the patient to the theatre and examine the patient under anesthesia. During the examination it was revealed that the lower half of the rectus sheath was fully gapped. At the lower $1 / 3^{\text {rd }}$ of the wound bowel was coming out through the rectus whereas in the upper wound the omentum was covering the bowel. The wound edges and the facial tissue were edematous and very friable.

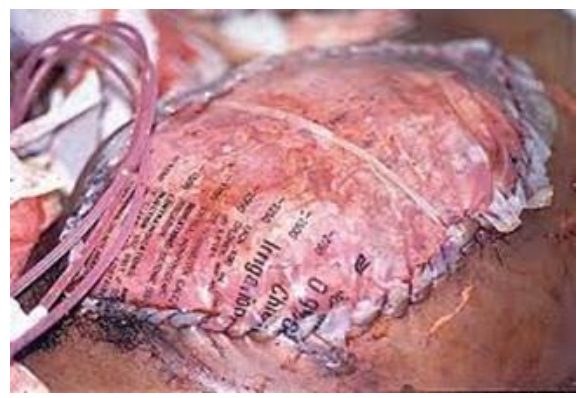

Figure 1 - Sterile urine bag to cover the wound

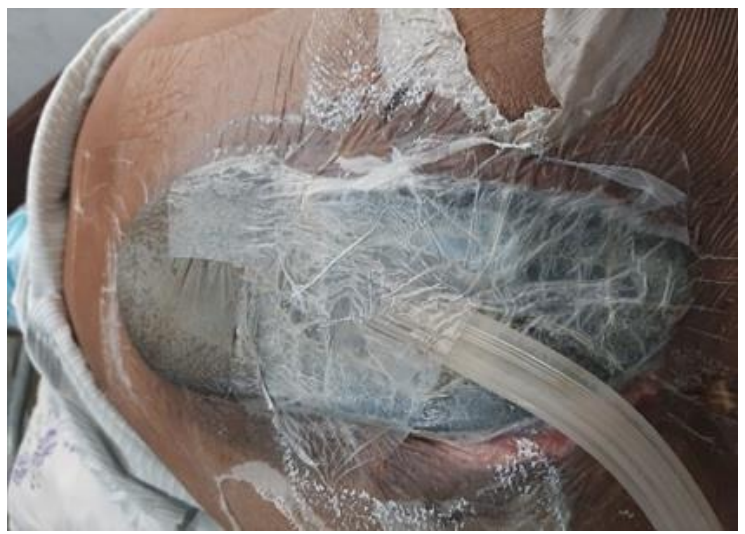

Figure 2 - Vacuum assisted closer (VAC) placement after 3 rd day of urine bag closer

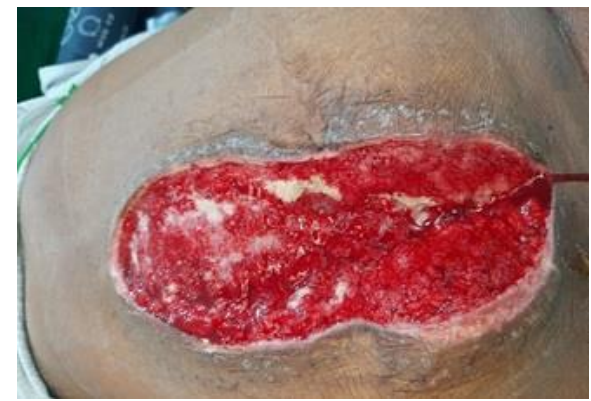

Figure 3 - 6th day of Vacuum assisted closer, after removing the VAC dressing

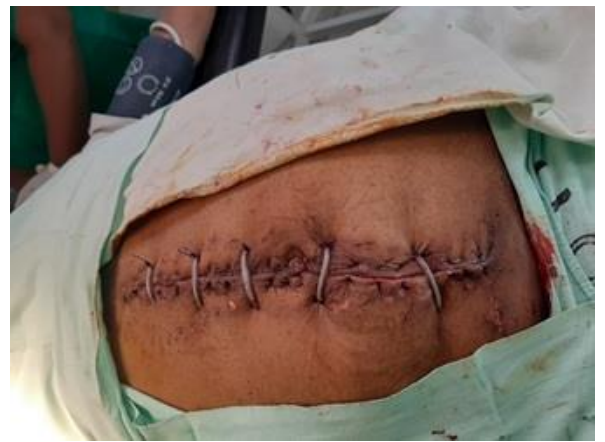

Figure 4 - Placement of tension sutures after removing the VAC dressing 
After necrotic wound edges and unhealthy facial tissue were debrided, it was noted that the wound gap was fairly big. Hence primary closure was not possible. In consequence we had to think of other means of temporary closure. There by applying the principles of Bogota bag theory we used a sterile urine bag to close the gap. The bag was anchored to the facial sheath with non-absorbable continuous stitch. The Intercostal tube was placed over the wound and taken out through a separate incision. It was connected to the close passive draining system. Three days later a thin layer of tissue was noted over the small bowel which was exposed at the lower end of the wound. As no more bowel loops were visible on the third day of Bogota bag (urine bag), Vacuum Assisted Closing (VAC) system was employed. On the sixth day of VAC, dressing was removed. There it was noted formation of adequate granulation tissue at the base and markedly reduced wall oedema. As a result, the wound edges were able to close without much tension. Hence the skin edges were closed with tension sutures and between tension sutures skin approximated with simple interrupted mattress sutures. Postoperatively the patient's parameters were quite normal. All the inflammatory markers were within normal range. Three days after applying the tension sutures the patient was discharged (that was 12 days after the burst abdomen). One week later the patient was reviewed in the surgical clinic and found to have no significant complaints. Interrupted sutures were removed on the 10th day after suturing. The tension sutures were removed on the 18th day of suturing.

\section{Discussion}

Surgical wound dehiscence (burst abdomen) is a complication following surgery in which the management makes a clinical challenge. Full thickness abdominal dehiscence is a major catastrophe and is dreaded by all surgeons. It has been noted that the incidence is increasing globally among aging populations and people with obesity, chronic diseases, immunocompromised conditions and patients with pulmonary condition etc. The risk factors for burst abdomen include local and systemic events which lead to poor wound healing. Some of them are elderly patients; more than 60 years, diabetes patients with blood sugar more than 140 $\mathrm{mg} / \mathrm{dL}$, malnutrition (hypoproteinemia- serum protein less than $6 \mathrm{~g} / \mathrm{dl}$ ), obesity-BMI more than 13 , anaemia-less than $10 \mathrm{~g} / \mathrm{dl}$ of hemoglobin, intra-abdominal malignancy, wound sepsis, operating time more than 2.5hr. ${ }^{5}$ Chronic pulmonary disease, emergency surgery are the other contributing factors. Pradeep G, et al [7] has shown in their study that malnutrition is a major risk factor. It accounts for $20.3 \%$ of burst abdomen followed by obesity $12.5 \%$. Anemia accounted for $12.4 \%$, wound infection $12.9 \%$, cough and abdominal distention $9.6 \%$ and $8.5 \%$ respectively. Amin et al

[8] in Pakistan has shown that a higher prevalence of burst abdomen is seen in emergency surgeries $(14.8 \%)$ than elective surgeries $(2.7 \%)$. Study done by Parmar et al [9] has shown that the maximum incidence of burst abdomen seen within 7 days. Due to the possible anatomical factors vertical upper abdominal wounds are more likely to burst. Disruption of segmental blood supply and denervation with vertical incision weaken the wound. On the other hand it damages the transversely running elastic fibers. Due to the seriousness it is of utmost important to prevent and manage burst abdomen in a rational manner. Key points to prevent and manage burst abdomen are,

1. Identify the risk factors and rectify if possible.

2. Identify the signs and symptoms of wound dehiscence. (Pain at the wound with serosanguinous discharge with acute inflammatory signs are pathognomonic).

3. Accurate assessment and categorize the type of wound dehiscence (whether it is a partial or full thickness).
4. See whether it is possible to close primarily. If not let it heal with secondary intentions. When we employ the second option it is crucial to prepare the wound bed in a good manner [4].

5. Supportive measures, control infection and optimize the comorbidity will speed up the recovery.

Burst abdomen could be managed effectively once we adhere to above principles.

Primary closure is the most popular practice unless there's no significant tissue tension. This is better in fit patients whose wound dehiscence is purely due to technical failure.[10] This method is not feasible when wound infection, bowel oedema and peritonitis is there. When primary closure is not possible open technique is the option where one must have healing wound edge by debridement of unhealthy tissue. Many techniques have been used to cover the defect temporarily or permanently. Mesh repair is one of the favorite methods where high complications such as enteric fistula has been noted. This could be minimized by placing absorbable mesh with interpose omentum [11] VAC currently is very popular where sterile sponge is placed over the defect and low continuous negative suction is applied [12]. This wound can be grafted or kept to heal with secondary intentions later. These patients will end up with incisional hernia. Bogota bag is a simple plastic bag used to cover the defect which is sutured to the wound edge facial tissue to allow free expansion of viscera preventing abdominal compartment syndrome. The bag could be changed every one to two weeks' time [13].

Our patient possible causes for wound dehiscence are

- Age - 62 years

- $\quad$ Morbid obesity - BMI-36

- Intra-abdominal malignancy - Sigmoid tumor with local infiltration

- $\quad$ Operating time More than $3 \mathrm{hr}$

- Wound infection - Culture positive (Staph)

- Delay in detection

Though it was noted that the patient was having an infection, dehiscence was apparent due to a very thick fat layer (more than $12 \mathrm{~cm}$ ). Even though the wound was more gaped and had discharged on the $7^{\text {th }}$ day, it was not possible to see bowel loops. However High degree of suspicious warrant to inspect the wound under anesthesia. During the examination under anesthesia the patient had a full thickness abdominal burst at the lower end of the wound.

During the wound exploration it was found to have an unhealthy wound edge with facial tissue necrosis with significant tissue oedema. The edematous unhealthy tissue which was under tension was not able to be brought together. Hence it was decided to manage the wound with open technique (Laparostomy). Mesh repair was not possible as we had no absorbable mesh. VAC placement was discouraged due to the lower end of the wounds' bowel not covered with any kind of tissue. Considering the necrotic edematous wound edge with morbid obesity and tissue tension it was decided to manage the patient with Bogota bag principle. There we used a sterile urine bag, which was sutured to the well debride facial edges. A 28-gage intercostal tube was placed on the wound and taken out through a separate stab so that it did not disturb the main wound. It was connected to the bag and left to drain in a passive manner. On the third day it was noted that the lower part of the wounds' bowel was covered with a thin layer of tissue. That led us to place the VAC system. On the 6th day of VAC, the patient was taken back to theatre and the wound was uncovered. There a good granulation tissue was noted. 
Because of the good granulation tissue rather than going for a second VAC we had two options.

1 Let it granulate and later skin graft.

2 Have the skin closed with tension suture.

Due to the thick layer of the subcutaneous fat tissue, it was assumed that it may take a longer time to fill up the gap. Therefore, we decide to go ahead with the second option where we approximated the skin with tension suture. Patient had a good recovery and was able to discharge on the $13^{\text {th }}$ day of his burst abdomen.

\section{Conclusion}

Burst abdomen is a serious sequel of impaired wound healing. Adherence to the proper technique and genuine effort to minimize the predisposing factors together with strict aseptic procedure play a much greater role in prevention of the event.

When the primary closure is not possible, it is better to use a combination of techniques depending on the wound stage. It helps us to send the patient back to society quite early.

\section{References}

1. Webster C, Neumayer L, (2003) Smout R et al. National Veterans Affairs Surgical Quality Improvement Program. Prognostic module of abdominal wound dehiscence after laparotomy. J Surg Res; 109: 130-137.

2. Gislason H, Gronbech JE, (1995) Soreide O. Bust abdomen and incisional hernia after major gastrointestinal operation comparison of three closure technique. Eur J Surg; 161:349-354.
3. Fleischer GM, Rennert A, (2000) Ruhmer M. infected abdominal wall and burst abdomen. Chirurg; 71: 754-62.

4. Kylie SH, Karen O, (2018) Elizabeth H. Ten top tips: Management of surgical wound dehiscence. Wound Asia; 1 (1) 1619.

5. Lakshmi G, Ravimohan TR. (2018) Post laparotomt abdominal wound dehiscence -A study in tertiary care hospital. International Journal of Contemporary Medical Research.; 5 (1): 77-83.

6. Pradeep Soni, Vibha Baghel Haripriya, Vishnu Dutt. (2015) Bust abdomen-A post- operative morbidity. International Journal of Scientific Study; 3 (6): 175-177.

7. Amini AQ, Kh an NA, Ahamad J, Memon AS. (1970) Management of abdominal wound dehiscence: still a challenge. Pak J Surg; 29: 84-7.

8. Parmar G, Gohil A, Hathila V. (2008) Burst abdomen: A grave postoperative complication. Internet J Surg; 20: 1-8.

9. Cliby W. (2002),Abdominal Incision Wound Breakdown. Cli Obstet Gynecol; 45(2):507-17.

10. Ghimenton F, Thomson SR, (2000) Muckart D, Burrows R. Abdominal content containment: practicalities and outcome. $\mathrm{Br} J$ Surg; 87(1): 106-9.

11. Paran H, Mayo A, (2001) Afanasiev A et al. Stage primary closure of the Abdominal wall in patient with Abdominal compartment Syndrom. J Trauma; 51(6) 1204-6.

12. Sukumar N, Shaharin S,RazmanJ,Jasmi AY. Bogota Bag in the Treatment of Abdominal Wound Dehiscence. Med J Malayasia 2004 59(2) 281-3.
This work is licensed under Creative Commons Attribution 4.0 License

To Submit Your Article Click Here: Submit Manuscript

DOI: $10.31579 / 2690-1897 / 091$

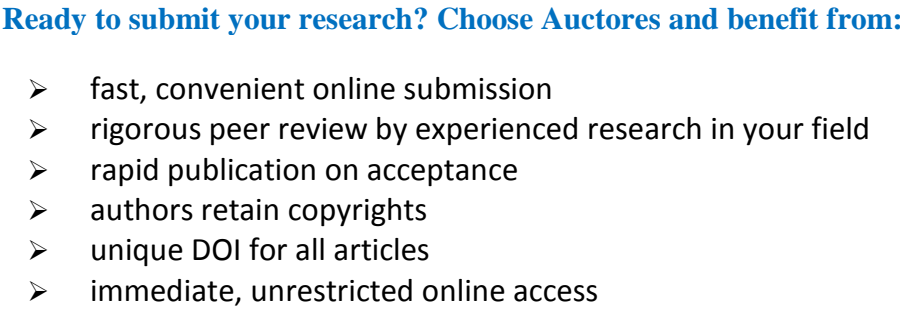

Ready to submit your research? Choose Auctores and benefit from:

> fast, convenient online submission

$>$ rigorous peer review by experienced research in your field

$>$ rapid publication on acceptance

$>$ authors retain copyrights

$>$ unique DOI for all articles

$>$ immediate, unrestricted online access

At Auctores, research is always in progress.

Learn more https://auctoresonline.org/journals/journal-of-surgical-casereports-and-images 